\title{
GUEST EDITORIAL \\ Placebo effects: a new paradigm and relevance to psychiatry
}

\author{
Daniel McQueen ${ }^{1}$ and Paul St John Smith ${ }^{2}$
}

Child and Adolescent Psychiatrist, Royal Free Hampstead NHS Trust, London, UK, email daniel.mcqueen@ virgin.net

${ }^{2}$ General Adult Psychiatrist Hertfordshire Partnership Foundation Trust, UK, email paulstjohnsmith@hotmail.

\begin{abstract}
Systematic evaluations show that placebo treatments can have large effects, sometimes larger than those of 'evidence-based treatments'. This is the 'efficacy paradox'. The neurobiology of placebo effects is being mapped out. Placebo effects are no less real or, in some illnesses, clinically important than the effects of direct biomechanical or pharmacological interventions. The technical model of medicine seeks impersonal technologies that can be applied independently of context and person. This approach has had spectacular success in the treatment of disease but meaning, cultural context, interpersonal effects, personal preferences and values are enormously important in the treatment of illness. The study of placebo reveals aspects of the biology of interpersonal relationships and the social environment. The evidence demonstrates that interpersonal healing (sometimes called placebo) in illness is just as real, scientific and biological as technological healing. This is a paradigm shift.
\end{abstract}

Paradigm shifts occur, according to Thomas Kuhn, when scientists encounter anomalies that cannot be explained by the existing, accepted paradigm within which scientific progress has hitherto been made.

Placebo effects have been a scientific curiosity for years, but tainted by associations with quackery and dishonesty. Recently, research on placebo effects has greatly increased. In June 2011 the Royal Society devoted a themed issue of its Philosophical Transactions to the placebo, which presented current understanding of psychobiological mechanisms, anomalies in placebo research, and how to harness placebo effects in clinical practice (Meissner et al, 2011).

Placebo effects may be simplistically defined as those accruing from taking dummy pills or inactive treatments. In placebo-controlled randomised controlled trials (PCRCTs) placebo is defined negatively, as those non-specific (typically nonpharmacological) effects to be subtracted from the treatment arm, to reveal the specific (typically pharmacological) effect. Here, placebo is 'noise' obscuring the 'signal' of 'real' treatment. Recently, placebo effects have been defined positively as the specific effects arising from caregiving.

Systematic evaluations reveal that placebo treatments can have large effects, sometimes larger than the effects of properly evaluated 'evidence- based treatments'. This is the 'efficacy paradox' (Kaptchuk et al, 2010). The neurobiology of placebo effects (nuclei, pathways, neurotransmitters, peptides and hormones) is being mapped out. There is evidence for various psychological mechanisms, including classical conditioning, evaluative conditioning, expectation (including the expectations of professionals), the quantity of care and attention received from professionals, and the quality of the therapeutic relationship or alliance (Meissner $e t a l$, 2011). Placebo effects are no less real or, in some illnesses, clinically important than the effects of direct biomechanical or pharmacological interventions.

Meta-analyses of PCRCTs demonstrate greater placebo responses for subjective symptoms, but far less for objectively measured physical parameters. Improvements also occur in no-treatment groups. This distinguishes technological healing (interventions acting directly on physical processes in the body, working even in unconscious patients), interpersonal healing (requiring a conscious patient to engage with symbolic interventions that influence perception, meaning and subjective experience) and natural healing (natural history of a disease, the body's natural responses to disease, and regression to the mean) (Miller et al, 2009). Healing rituals occur in all human societies. The biological substrate and instinctual underpinning of interpersonal healing are likely to be rooted in the evolution by natural selection of mammalian attachment instincts and related grooming (bonding) behaviours. The investigation of placebo effects and mechanisms has emerged as a way of studying the 'healing situation'.

The technological model of medicine seeks impersonal means of cure that can be applied independently of context and person. The PCRCT is a central tool of technological medicine. It developed precisely to control for interpersonal healing effects and individual and contextual factors. This approach has had spectacular success in the treatment of disease (the objective anatomicopathophysiology). However, meaning, cultural context, interpersonal effects, personal preferences and values are enormously important in the treatment of illness (the phenomenological subjective experience), particularly psychiatric conditions (Miller et al, 2009).

The size of a placebo effect is highly dependent on the conditions of treatment, specifically the person's active participation, beliefs, preferences and the quality of relationships with clinicians. Lidstone et al (2010) manipulated the expectation of patients with Parkinson's disease that they 
were receiving placebo and found that significant dopamine release occurred when the declared probability of receiving active medication was $75 \%$, but not at lower probabilities. Manipulating treating clinicians' beliefs in the efficacy of treatment also leads to significant differences in patient outcomes.

Some drugs may exert their effect by amplifying placebo responses. Benedetti et al (1995) showed that the cholecystokinin antagonist proglumide was more effective in reducing postoperative pain than placebo, which was more effective than no treatment (cholecystokinin opposes endogenous opiate pathways). However, when proglumide was given covertly it had no effect. The authors concluded that proglumide has no direct effect on pain pathways, but instead potentiates a placeboactivated endogenous opiate system, and therefore is effective only when combined with the placebo mechanisms inherent in the clinical encounter. Similar studies using covert administration of drugs indicate a far larger role for placebo effects than has hitherto been recognised (Benedetti et al, 2003). The finding that some drugs exert effects by acting on pathways that are activated in placebo responses, if replicated, further complicates the simple 'placebo $v$. specific effect' dichotomy.

Understanding the evolution of primary emotional systems reveals that the critical determinants of affects, psychiatric disorders and placebo effects are interpersonal relationships and the social environment. Recognition of the importance of relationships has focused interest on the 'art of medicine' and the informal psychotherapeutic processes that occur between skilled empathic clinicians and their patients, which can help patients to achieve more successful ways of coping with illness, disease and life's other challenges. Psychotherapy can be seen as a pure form of the doctor-patient relationship, stripped of pharmacological effects. It has been argued that psychotherapy is analogous to a chemotherapy placebo, or even that psychotherapy is only placebo. However, this is derogatory to both psychotherapy and placebo. What does the psychotherapy literature reveal about interpersonal processes leading to therapeutic change? Different bona fide shortterm psychotherapies, be they psychoanalytic, behavioural, cognitive, humanistic, or integrative, have globally comparable outcomes across a range of conditions, with effect sizes in the region of 0.85 . This is the 'equivalence paradox'. Claims for the effectiveness of specific techniques have been largely explained by strong biases in investigator allegiance. Psychotherapy process research reveals that specific techniques account for very little of the variance in outcome, far less than the so-called 'non-specific' effects of being in therapy. Non-specific factors can be conceptualised in various ways but generally include: the therapeutic alliance (consistently accounting for most of the variance in outcome), patient factors (such as engagement), therapeutic focus (having a specific focus leads to better outcome), expectation of a good outcome (clinicians' and patients' expectations of success tend to be self-fulfilling), and patient and therapist characteristics (Messer \& Wampold, 2002).

In the pharmacological treatment of depression three-quarters of the effects of antidepressants are achieved by placebo. Up to one-quarter of improvements may be due to natural history and half to 'true' placebo effects (Kirsch \& Sapirstein, 1998). Placebo effects may be smaller and pharmacological effects proportionately larger as severity increases (Kirsch et al, 2008). McKay et al (2006) reanalysed data from the US National Institute of Mental Health's Treatment of Depression Collaborative Research Program and showed that the contribution of the therapeutic alliance outweighed the modality of treatment, whether cognitivebehavioural therapy (CBT), interpersonal therapy (IPT), imipramine or placebo. Individual psychiatrist effects accounted for more variance in outcome than did the difference between taking imipramine or placebo $(9.1 \%$ v. $3.4 \%$ of variance in score on the Beck Depression Inventory). The most effective psychiatrists were responsible for the largest clinical improvements irrespective of whether their patients were taking placebo or medication. The most effective psychiatrists had better outcomes with placebo than the least effective psychiatrists had with antidepressants.

Placebo responses are less well studied in schizophrenia, but may be similar to those in depression (Kinon et al, 2011). However, rates of dismissive and unresolved attachment in schizophrenia are at least double those in depression; this complicates relationships with clinicians and therefore interpersonal healing (Dozier et al, 1999).

Further support for the importance of interpersonal healing comes from the mental health recovery movement, which has highlighted the importance of hope, validation, supportive relationships, engagement, coping skills and meaning. These factors are central to both placebo and psychotherapy.

Prescribing evidence-based treatments and simply expecting the technology to work while failing to establish therapeutic relationships profoundly limits clinical effectiveness. In the absence of long-term research on placebo effects, evidence from psychotherapy process research suggests that relationship factors promote therapeutic change. The ability to form therapeutic relationships should be promoted in the training of psychiatrists. A range of strategies may be required, including: training in communication skills, reflective practice (Balint groups and work discussion groups) and conducting psychotherapeutic treatments under supervision.

The study of placebo reveals aspects of the biology of interpersonal relationships and the social environment. The evidence demonstrates that interpersonal healing (sometimes called placebo) in illness is just as real, scientific and biological as technological healing. This is a paradigm shift. 


\section{References}

Benedetti, F., Amanzio, M. \& Maggi, G. (1995) Potentiation of placebo analgesia by proglumide. Lancet, 346, 1231.

Benedetti, F., Maggi, G., Lopiano, L., et al (2003) Open versus hidden medical treatments: the patient's knowledge about a therapy affects the therapy outcome. Prevention and Treatment, 6 , ArtID 1a (no pagination).

Dozier, M., Stovall, K. C. \& Albus, K. E. (1999) Attachment and psychopathology in adulthood. In Handbook of Attachment: Theory, Research, and Clinical Applications (eds J. Cassidy \& P. R. Shaver), pp. 497-519. Guilford Press.

Kaptchuk, T. J., Friedlander, E., Kelley, J. M., et al (2010) Placebos without deception: a randomised controlled trial in irritable bowel syndrome. PLOS ONE, 5(12), e15591.

Kinon, B. J., Potts, A. J. \& Watson, S. B. (2011) Placebo response in clinical trials with schizophrenia patients. Current Opinion in Psychiatry, 24, 107-113.

Kirsch, I. \& Sapirstein, G. (1998) Listening to Prozac but hearing placebo: a meta-analysis of antidepressant medication. Prevention and Treatment, 1, 1-17.
Kirsch, I., Deacon, B. J., Huedo-Medina, T. B., et al (2008) Initial severity and antidepressant benefits: a meta-analysis of data submitted to the Food and Drug Administration. PLoS Medicine, $5(2), e 45$

Lidstone, S. C., Schulzer, M., Dinelle, K., et al (2010) Effects of expectation on placebo-induced dopamine release in Parkinson disease. Archives of General Psychiatry, 67, 857-865.

McKay, K. M., Imel, Z. E. \& Wampold, B. E. (2006) Psychiatrist effects in the psychopharmacological treatment of depression. Journal of Affective Disorders, 92, 287-290.

Meissner, K., Kohls, N. \& Colloca, L. (eds) (2011) 'Placebo effects in medicine: mechanisms and clinical implications'. Theme issue of Philosophical Transactions of the Royal Society B, 366 , 1781-1930.

Messer, S. B. \& Wampold, B. E. (2002) Let's face facts: common factors are more potent than specific therapy ingredients. Clinical Psychology: Science and Practice, 9, 21-25.

Miller, F. G., Colloca, L. \& Kaptchuk, T. J. (2009) The placebo effect: illness and interpersonal healing. Perspectives in Biology and Medicine, 52, 518-539.

\section{THEMATIC PAPERS}

Behavioural and Brain Sciences Unit, Institute of Child Health, London, UK, email dskuse@ich. ucl.ac.uk

\section{Recovery}

\author{
David Skuse
}

The concept of 'recovery' as applied to severe mental illness has fostered a cultural change in attitudes to the long-term outcome of conditions such as schizophrenia. 'Recovery' has a specific meaning in this context. It refers to the possibility that even in the presence of a chronic psychiatric disorder there is hope for a life that has value. The affected individual can still make a contribution to society; he or she can expect to live independently and with dignity. The term implies that our traditional medical model of illness lacks the longer-term perspective on how patients might learn to cope with their condition.

We present three themed articles on 'recovery' as applied to mental healthcare. Two of those articles review the concept of recovery and its historical antecedents. The third concerns the specific case of schizophrenia and reviews surprising findings about the prognosis of the condition in different countries and cultures.

We start with an authoritative account of the origins of the 'recovery movement' in the USA, from Anthony Ahmed and colleagues. Back in the early 1970s grave concern was being expressed by patients, their families and some professionals about the management of severe mental illness and the role played at that time by lifelong institutional care, which was then prevalent in the USA and many other high-income countries. As a reaction against this management strategy there was a push for greater patient participation in decision-making. This was the start of the focus on 'recovery'. It involved the establishment of advocacy for those who wished to leave institutional care and make a future for themselves within rather than outside the community at large. Such a movement challenged societal stigma about those with impaired mental health.

Ahmed and colleagues discuss the development of the 'recovery theme' of psychiatric illness in the USA, where it appears to have been most enthusiastically supported. In their view, in the UK we are lagging behind. On the other hand, Jed Boardman and Geoff Shepherd are optimistic about the changes that we are beginning to see here. They discuss in outline the Implementing Recovery Organisational Change project, which is a national strategy that aims to help organisations in the UK become more supportive of recovery.

Finally, Aleksandar Janca and Sivasankaran Balaratnasingam take a historical perspective on cross-national comparisons of prognosis in schizophrenia. Since the pioneering projects devised by John Wing in collaboration with the World Health Organization, 40 years ago, evidence has been accumulating that indicates there are major international differences in the prevalence and the prognosis of schizophrenia. Here, the surprising and controversial accounts of better 'recovery' from the condition in countries that have rudimentary mental health services, than in the psychiatrist-led, medication-oriented, hospital environments of the Western world, are debated. 